\title{
On Infinite Prefix Normal Words
}

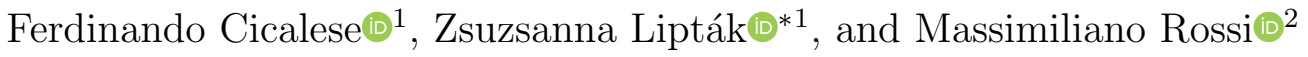 \\ ${ }^{1}$ Department of Computer Science, University of Verona, Verona, Italy \\ ${ }^{2}$ Department of Computer and Information Science and Engineering, University \\ of Florida, Gainesville, FL, United States \\ ${ }^{1}\left\{\right.$ ferdinando.cicalese, zsuzsanna.liptak\}@univr.it, ${ }^{2}$ rossi.m@ufl.edu
}

Article published in Theoretical Computer Science (2021)

doi: $10.1016 /$ j.tcs.2021.01.015

\begin{abstract}
Prefix normal words are binary words with the property that no factor has more 1s than the prefix of the same length. Finite prefix normal words were introduced in [Fici and Lipták, DLT 2011]. In this paper, we study infinite prefix normal words and explore their relationship to some known classes of infinite binary words. In particular, we establish a connection between prefix normal words and Sturmian words, between prefix normal words and abelian complexity, and between prefix normality and lexicographic order 1
\end{abstract}

Keywords - combinatorics on words, prefix normal words, infinite words, Sturmian words, abelian complexity, paperfolding word, Thue-Morse sequence, lexicographic order

\section{Introduction}

Prefix normal words are binary words where no factor has more 1s than the prefix of the same length. As an example, the word 11100110101 is prefix normal, while 11100110110 is not, since it has a factor of length 5 with four 1s, while the prefix of length 5 has only three 1s. Finite prefix normal words were introduced in [18, and further studied in [10, 11, 31, 14, 3, 19, 9].

One motivation for studying prefix normal words comes from the problem of Indexed Binary Jumbled Pattern Matching [7, 8, 25, 21, 2, 20, 13, 16, 1]: Given a finite word $s$ of length $n$, construct an index in such a way that the following type of queries can be answered efficiently: for two integers $x, y \geq 0$, does $s$ have a factor with $x$ 1s and $y 0$ s? As shown in [18, 11, prefix normal words can be used for constructing such an index, via so-called prefix normal forms.

Prefix normal words have also been shown to form bubble languages [29, 30, 10], a family of binary languages with efficiently generable combinatorial Gray codes; the language of prefix normal words has connections to the Binary Reflected Gray Code [31; and, recently, prefix normal words also appeared in a graph theoretic context [6]. Indeed, three sequences related to

* Corresponding author

${ }^{1}$ This is an extended version of our paper presented at SOFSEM 2019 [15. 
prefix normal words are present in the On-Line Encyclopedia of Integer Sequences (OEIS 33]): A194850 (the number of prefix normal words of length $n$ ), A238109 (a list of prefix normal words over the alphabet $\{1,2\}$ ), and A238110 (maximal equivalence class sizes of words with the same prefix normal form).

In [14, we introduced infinite prefix normal words and analyzed a particular procedure that, given a finite prefix normal word, extends it while preserving the prefix normality property. We showed that the resulting infinite word is ultimately periodic. In this paper, we present a more comprehensive study of infinite prefix normal words, covering several classes of known and well studied infinite words. We will now give a quick tour of the paper (for precise definitions, see Section 2).

\subsection{Our results}

One way of obtaining infinite prefix normal words is by extending finite prefix normal words. We specify two such operations which, in the limit, produce prefix normal words that are extremal with respect to density (Theorem 1 ).

There exist periodic, ultimately periodic, and aperiodic infinite prefix normal words: for example, the periodic words $0^{\omega}, 1^{\omega}$, and $(10)^{\omega}$ are prefix normal; the ultimately periodic word $1(10)^{\omega}$ is prefix normal; and so is the aperiodic word $10100100010000 \cdots=\lim _{n \rightarrow \infty} 1010^{2} \cdots 10^{n}$. The best studied class of aperiodic words are Sturmian words. We show that a Sturmian word $w$ is prefix normal if and only if $w=1 c_{\alpha}$ for some $\alpha$, where $c_{\alpha}$ is the characteristic word of slope $\alpha$ (Theorem 2).

We show further that every Sturmian word $w$ can be turned into a prefix normal word by prepending a fixed number of $1 \mathrm{~s}$, which only depends on the slope of $w$. This follows from a more general result regarding $c$-balanced words (Lemma 5. For example, the Fibonacci word

$$
\mathfrak{f}=0100101001001010010100100101001001 \cdots
$$

is not prefix normal, but the word $1 \mathfrak{f}$ is. Two other well-studied aperiodic words are the ThueMorse word and the Champernowne word. The Thue-Morse word

$$
\mathbf{t}=01101001100101101001011001101001 \cdots
$$

is not prefix normal but it can be turned into a prefix normal word by prepending two 1s: $11 \mathrm{t}$ is prefix normal. On the other hand, the binary Champernowne word

$$
\mathfrak{c}=0110111001011101111000100110101011 \cdots
$$

which is constructed by concatenating the binary expansions of the integers in ascending order, is not prefix normal and cannot be turned into a prefix normal word by prepending a finite number of $1 \mathrm{~s}$.

We also show that the notion of prefix normal forms from [18, 11] can be extended to infinite words. These can be used, similarly to the finite case, to encode the abelian complexity of the original word. The study of abelian complexity of infinite words was initiated in [27, and continued e.g. in [24, 4, 34, 12, 22. We establish a close relationship between the abelian complexity and the prefix normal forms of $w$ (Theorem 3). We demonstrate how this close connection can be used to derive results about the prefix normal forms of a word $w$. In some cases, such as for Sturmian words and words which are morphic images under the Thue-Morse morphism, we are able to explicitly give the prefix normal forms of the word (Corollary 3 and Theorem 5). Conversely, knowing its prefix normal forms allows us to derive results about the 
abelian complexity of a word. We also show how to compute the prefix normal forms of words that are binary uniform morphisms, based on an algorithm from [5] for computing their abelian complexity.

Another class of well-known binary words are Lyndon words. Notice that the prefix normal condition is different from the Lyndon condition 2 , for finite words, there are words which are both Lyndon and prefix normal (e.g. 110010), words which are Lyndon but not prefix normal (11100110110), words which are prefix normal but not Lyndon (110101), and words which are neither (101100). We study infinite prefix normal words and their prefix normal forms in the context of lexicographic orderings, and compare them to infinite Lyndon words 32 and the maxand min-words of [26] (Corollary 5).

Finally, we give conditions for periodicity and ultimate periodicity of prefix normal words in terms of their minimum density, a parameter introduced in [14] (Theorem 8).

\subsection{Overview of paper}

The paper is organized as follows. In Section 2, we introduce our terminology and give some simple facts about prefix normal words. In Section 3, we compare different operations that generate infinite prefix normal words by extending finite prefix normal words. In Section 4 , we study the relationship between Sturmian words and prefix normal words. Section 5 deals with the connection between prefix normality and abelian complexity, and Section 6 focuses on the relationship with lexicographic order. Finally, in Section 7, we analyze the relationship between periodicity and minimum density of prefix normal words.

\section{Basics}

In our definitions and notations, we follow mostly [23. A finite (resp. infinite) binary word $w$ is a finite (resp. infinite) sequence of elements from $\{0,1\}$. Thus an infinite word is a mapping $w: \mathbb{N} \rightarrow\{0,1\}$, where $\mathbb{N}$ denotes the set of positive integers. We denote the $i$ th character of $w$ by $w_{i}$. Note that we index words starting from 1 . If $w$ is finite, then its length is denoted by $|w|$. The empty word, denoted $\varepsilon$, is the unique word of length 0 . The set of binary words of length $n$ is denoted by $\{0,1\}^{n}$, the set of all finite words by $\{0,1\}^{*}=\cup_{n \geq 0}\{0,1\}^{n}$, and the set of infinite binary words by $\{0,1\}^{\omega}$. For a finite word $u=u_{1} \cdots u_{n}$, we write $u^{\text {rev }}=u_{n} \cdots u_{1}$ for the reverse of $u$, and for a finite or infinite word $u, \bar{u}=\bar{u}_{1} \bar{u}_{2} \cdots$ for the complement of $u$, where $\bar{a}=1-a$ for $a \in\{0,1\}$.

For two words $u, v$, where $u$ is finite and $v$ is finite or infinite, we write $u v$ for their concatenation. If $w=u x v$, then $u$ is called a prefix, $x$ a factor (or substring), and $v$ a suffix of $w$. We denote the set of factors of $w$ by $F c t(w)$ and its prefix of length $i$ by $\operatorname{pref}_{w}(i)$, where $\operatorname{pref}_{w}(0)=\varepsilon$. For a finite word $u$, we write $|u|_{1}$ for the number of $1 \mathrm{~s}$, and $|u|_{0}$ for the number of $0 \mathrm{~s}$ in $u$, and refer to $|u|_{1}$ as the weight of $u$. The Parikh vector of $u$ is $p v(u)=\left(|u|_{0},|u|_{1}\right)$. A word $w$ is called balanced if for all $u, v \in F c t(w),|u|=|v|$ implies $\left.|| u\right|_{1}-|v|_{1} \mid \leq 1$, and c-balanced if $|u|=|v|$ implies $\left.|| u\right|_{1}-|v|_{1} \mid \leq c$.

For an integer $k \geq 1$ and $u \in\{0,1\}^{n}, u^{k}$ denotes the $k n$-length word $u u u \cdots u(k$-fold concatenation of $u$ ) and $u^{\omega}$ the infinite word $u u u \cdots$. An infinite word $w$ is called periodic if $w=u^{\omega}$ for some non-empty word $u$, and ultimately periodic if it can be written as $w=v u^{\omega}$ for some $v$ and non-empty $u$. A word that is neither periodic nor ultimately periodic is called

\footnotetext{
${ }^{2}$ For ease of presentation, we are using Lyndon to mean lexicographically greatest among its conjugates; this is equivalent to the usual definition up to renaming characters.
} 
aperiodic. We set $0<1$ and denote by $\leq_{\text {lex }}$ the lexicographic order between words, i.e. $u \leq_{\operatorname{lex}} v$ if $u$ is a prefix of $v$ or there is an index $i \geq 1$ s.t. $\operatorname{pref}_{u}(i-1)=\operatorname{pref}_{v}(i-1)$ and $u_{i}<v_{i}$.

For an operation op $:\{0,1\}^{*} \rightarrow\{0,1\}^{*}$, we denote by op ${ }^{(i)}$ the $i$ th iteration of op. Further, let op* $(w)=\left\{\mathrm{op}^{(i)}(w) \mid i \geq 1\right\}$ and $\mathrm{op}^{\omega}(w)=\lim _{i \rightarrow \infty} \mathrm{op}^{(i)}(w)$, if it exists.

A binary morphism $\mu$ is a function $\mu:\{0,1\}^{*} \rightarrow\{0,1\}^{*}$ such that for all $u, v \in\{0,1\}^{*}$, $\mu(u v)=\mu(u) \mu(v)$. A binary morphism $\mu$ is called uniform if $|\mu(0)|=|\mu(1)|$. A fix point of a morphism $\mu$ is an infinite word $v$ such that $v=\mu^{\omega}(a)$ for some $a \in\{0,1\}$.

Definition 1 Let $w$ be a (finite or infinite) binary word. We define the following functions:

- $P_{w}(i)=\left|\operatorname{pref}_{w}(i)\right|_{1}$, the weight of the prefix of length $i$,

- $D_{w}(i)=P_{w}(i) / i$, the density of the prefix of length $i$,

- $F_{w}^{1}(i)=\max \left\{|u|_{1}: u \in F c t(w),|u|=i\right\}$ the maximum number of 1 s in a factor of length $i$,

- $f_{w}^{1}(i)=\min \left\{|u|_{1}: u \in F c t(w),|u|=i\right\}$, the minimum number of 1 s in a factor of length $i$,

- $F_{w}^{0}(i)=\max \left\{|u|_{0}: u \in F c t(w),|u|=i\right\}$, the maximum number of $0 s$ in a factor of length $i$,

- $f_{w}^{0}(i)=\min \left\{|u|_{0}: u \in F c t(w),|u|=i\right\}$, the minimum number of $0 s$ in a factor of length $i$.

Note that in the context of succinct indexing, the function $P_{w}(i)$ is often called $\operatorname{rank}_{1}(w, i)$. We are now ready to define prefix normal words.

Definition 2 (Prefix normal words) A (infinite or finite) binary word $w$ is called 1-prefix normal, or simply prefix normal, if $P_{w}(i)=F_{w}^{1}(i)$ for all $i \geq 1$ (for all $1 \leq i \leq|w|$ if $w$ is finite). It is called 0-prefix normal if $i-P_{w}(i)=F_{w}^{0}(i)$ for all $i \geq 1$ (for all $1 \leq i \leq|w|$ if $w$ is finite). We denote the set of all finite 1-prefix normal words by $\mathcal{L}_{\mathrm{fin}}$, the set of all infinite 1-prefix normal words by $\mathcal{L}_{\text {inf }}$, and $\mathcal{L}=\mathcal{L}_{\text {fin }} \cup \mathcal{L}_{\text {inf }}$.

In other words, a word is prefix normal if no factor has more 1s than the prefix of the same length. Given a binary word $w$, we say that a factor $u$ of $w$ satisfies the prefix normal condition if $|u|_{1} \leq P_{w}(|u|)$.

Example 1 The word 110100110110 is not prefix normal since the factor 11011 has four 1 s, which is more than in the prefix 11010 of length 5. The word 110100110010, on the other hand, is prefix normal. The infinite word (11001) ${ }^{\omega}$ is not prefix normal, because it has 111 as a factor, which has more 1 s than the prefix of length 3, but the word $(11010)^{\omega}$ is.

The following facts about infinite prefix normal words are immediate.

Lemma 1 1. For all $u \in \mathcal{L}_{\text {fin }}$, the word $w=u 0^{\omega} \in \mathcal{L}_{\text {inf }}$.

2. Let $w \in\{0,1\}^{\omega}$. Then $w \in \mathcal{L}$ if and only if for all $i \geq 1 \operatorname{pref}_{w}(i) \in \mathcal{L}$.

Definition 3 (Minimum density, minimum-density prefix, slope) Let $w \in\{0,1\}^{*} \cup\{0,1\}^{\omega}$. Define the minimum density of $w$ as $\delta(w)=\inf \left\{D_{w}(i) \mid 1 \leq i\right\}$. If this infimum is attained somewhere, then we also define $\iota(w)=\min \left\{j \geq 1 \mid \forall i: D_{w}(j) \leq D_{w}(i)\right\}$ and $\kappa(w)=P_{w}(\iota(w))$. We refer to $\operatorname{pref}_{w}(\iota(w))$ as the minimum-density prefix, the shortest prefix with density $\delta(w)$. For an infinite word $w$, we define the slope of $w$ as $\lim _{i \rightarrow \infty} D_{w}(i)$, if this limit exists. 
Remark 1 Note that $\iota(w)$ is always defined for finite words, while for infinite words, a prefix which attains the infimum may or may not exist. We note further that density and slope of infinite binary words do not necessarily coincide. In particular, while $\delta(w)$ exists for every $w$, the limit $\lim _{i \rightarrow \infty} D_{w}(i)$ may not exist, i.e., $w$ may or may not have a slope. As an example, consider the word $w=v_{0} v_{1} v_{2} \cdots$, where for each $i, v_{i}=1^{2^{i}} 0^{2^{i}}$. Then, $\delta(w)=1 / 2$ and $\lim _{i \rightarrow \infty} D_{w}(i)$ does not exist, since $D_{w}(i)$ has an infinite subsequence which is constant $1 / 2$, and another which tends to $2 / 3$.

Moreover, even for words $w$ for which the slope is defined, this can be different from the minimum density. If $w$ has slope $\alpha$, then $\alpha=\delta(w)$ if and only if for all $i, D_{w}(i) \geq \alpha$. For instance, the infinite word $01^{\omega}$ has slope 1 but its minimum density is 0 . On the other hand, the infinite word $1(10)^{\omega}$ has both slope and minimum density $1 / 2$.

\section{Operations generating infinite prefix normal words}

In [14, we introduced an operation which takes a finite prefix normal word $w$ ending in 1 and extends it by a run of 0 s followed by a new 1, in such a way that this new 1 is placed in the first possible position without violating prefix normality. This operation, called flipext, leaves the minimum density invariant. Moreover, by repeatedly applying the flipext operation, an infinite prefix normal word is produced which is the densest among all prefix normal words with given prefix $w$.

Here we extend the definition of flipext to all prefix normal words containing at least one 1 and show that the same properties hold, even if the original word $w$ does not end in 1 .

Definition 4 (Operation flipext) Let $w \in \mathcal{L}_{\text {fin }} \backslash\{0\}^{*}$. Define flipext $(w)$ as the finite word $w 0^{k} 1$, where $k=\min \left\{j \mid w 0^{j} 1 \in \mathcal{L}\right\}$. We further define the infinite word $v=$ flipext $^{\omega}(w)$.

The next proposition is a slightly more general form of Lemma 13 from [14]:

Proposition 1 Let $w \in \mathcal{L}_{\text {fin }} \backslash\{0\}^{*}$ and $v \in$ flipext $^{*}(w) \cup\left\{\right.$ flipext $\left.^{\omega}(w)\right\}$. Then $\delta(v)=\delta(w)$, and, as a consequence, $\iota(v)=\iota(w)$ and $\kappa(v)=\kappa(w)$. Moreover, $D_{v}(j \cdot \iota(w))=\delta(w)$ for all $j \geq 1$.

Proof. Let $w \in \mathcal{L}$. If the last character of $w$ is a 1 , then the claim holds by Lemma 13 of [14.

Else $w$ ends in a run of 0s. Let $\ell$ be the length of this run, and $w^{\prime}$ be such that $w=w^{\prime} 0^{\ell}$. Let $w^{\prime \prime}=\operatorname{flipext}\left(w^{\prime}\right)=w^{\prime} 0^{k} 1$, i.e. by definition of flipext, $k$ is minimal s.t. $w^{\prime} 0^{k} 1 \in \mathcal{L}$. If $\ell \leq k$, then flipext $(w)=\operatorname{flipext}\left(w^{\prime}\right)=w^{\prime \prime}$. Since $w^{\prime}$ is a prefix of $w$, and $w$ is a prefix of $w^{\prime \prime}$, we have $\delta\left(w^{\prime}\right) \geq \delta(w) \geq \delta\left(w^{\prime \prime}\right)$. Since $w^{\prime}$ ends in a $1, \delta\left(w^{\prime \prime}\right)=\delta\left(w^{\prime}\right)$, and thus $\delta\left(w^{\prime \prime}\right)=\delta(w)$.

Otherwise $\ell>k$, therefore flipext $\left(w^{\prime}\right)=w^{\prime} 0^{\ell^{\prime}} 1 \in \mathcal{L}_{\text {fin }}$ for some $\ell^{\prime}<\ell$, hence $w^{\prime} 0^{\ell} 1 \in \mathcal{L}_{\text {fin }}$. The latter implies flipext $(w)=w 1$ and $\delta(\operatorname{flipext}(w))=\delta(w)$.

Further iterations flipext ${ }^{(i)}(w)$ fulfil the claim due to the fact that flipext $(w)$ ends in a 1.

We now show the second statement: $D_{v}(j \cdot \iota(w))=\delta(w)$ for all $j \geq 1$. We show it by induction. It is clearly true for $j=1$, moreover for each $j>1$ assuming $D_{v}((j-1) \cdot \iota(w))=\delta(w)$ and letting $w^{\prime}=\operatorname{pref}_{v}((j-1) \cdot \iota(w))$ and $w^{\prime \prime}$ be the substring of size $\iota(w)$ such that $w^{\prime} w^{\prime \prime}=\operatorname{pref}_{v}(j \cdot \iota(w))$, we have

$$
\begin{aligned}
\delta(w) & =\delta(v) \leq D_{v}(j \cdot \iota(w))=\frac{\left|w^{\prime}\right|_{1}+\left|w^{\prime \prime}\right|_{1}}{j \cdot \iota(w)} \\
& \leq \frac{P_{w}(\iota(w))(j-1)+P_{w}(\iota(w))}{j \cdot \iota(w)}=\frac{P_{w}(\iota(w))}{\iota(w)}=\delta(w),
\end{aligned}
$$


where in the second inequality we are using $\left|w^{\prime}\right|_{1}=P_{w}(\iota(w))(j-1) \iota(w)$ (induction hypothesis) and $\left|w^{\prime \prime}\right|_{1} \leq P_{w}(\iota(w))$ (since $v$ is prefix normal).

The next proposition states that the infinite word which is generated by repeatedly applying the flipext operation is the densest among all prefix normal words with prefix $w$.

Proposition 2 Let $w \in \mathcal{L}_{\text {fin }} \backslash 0^{*}, v=$ flipext $^{\omega}(w)$, and let $z \in \mathcal{L}_{\text {inf }}$ such that $\operatorname{pref}_{z}(|w|)=w$. Then for every $i=1,2, \ldots$ we have $P_{v}(i) \geq P_{z}(i)$.

Proof. We argue by contradiction. Let $i$ be the smallest integer such that $P_{v}(i)<P_{z}(i)$. Clearly $i>|w|$ and, by the minimality assumption we must have $P_{v}(i-1)=P_{z}(i-1)$ and $v_{i}=0, z_{i}=1$. By definition of flipext there must exist $j<i$ such that $\left|v_{j+1} \ldots v_{i-1} 1\right|_{1}>P_{v}(i-j) \geq P_{z}(i-j)$, for otherwise we would have $v_{i}=1$. Since $v$ is prefix normal, it also follows that we have $\left|v_{j+1} \ldots v_{i-1} v_{i}\right|_{1}=P_{v}(i-j) \geq P_{z}(i-j)$.

From this, since by the minimality of $i$ it holds that $P_{z}(j) \leq P_{v}(j)$, we have that $\left|z_{j+1} \ldots z_{i-1} z_{i}\right|_{1}=$ $P_{z}(i)-P_{z}(j)>P_{v}(i)-P_{v}(j)=P_{v}(i-j) \geq P_{z}(i-j)$, violating the prefix normality of $z$.

We now define a different operation, called lazy-flipext, which, given a prefix normal word $w$, extends it by adding $0 \mathrm{~s}$ as long as the minimum density of the resulting word is not smaller than $\delta(w)$, and only then adding a 1 . We show that this operation preserves the prefix normality of the resulting word.

Definition 5 (Operation lazy-flipext) Let $\alpha \in(0,1]$ and let $w \in \mathcal{L}_{\text {fin }}$ with $\delta(w) \geq \alpha$. We define lazy-flipext $(w, \alpha)$ as the finite word $w 0^{k} 1$ where $k=\max \left\{j \mid \delta\left(w 0^{j}\right) \geq \alpha\right\}$. We further define the infinite word $v=$ lazy-flipext ${ }^{\omega}(w, \alpha)$.

Example 2 Let $w=111$ and let $\alpha=\sqrt{2}-1$. Then lazy-flipext $(w, \alpha)=11100001$, since $\delta(1110000)=3 / 7 \geq \alpha$ and $\delta(11100000)=3 / 8<\alpha$. Further, lazy-flipext ${ }^{(2)}(w, \alpha)=1110000101$, since $\delta(111000010)=4 / 9 \geq \alpha$ and $\delta(1110000100)=2 / 5<\alpha$.

Lemma 2 Let $\alpha \in(0,1]$. For every $w \in \mathcal{L}_{\text {fin }}$ with $\delta(w) \geq \alpha$, the word $v=$ lazy-flipext $(w, \alpha)$ is also prefix normal, with $\delta(v) \geq \alpha$.

Proof. First note that $\delta(v) \geq \alpha$ by definition. Now write $v=w 0^{k} 1$, and let $u=\operatorname{flipext}(w)=$ $w 0^{\ell} 1$. Recall that $\ell=\min \left\{\bar{j} \mid w 0^{j} 1 \in \mathcal{L}\right\}$. If $k<\ell$, this implies $\delta(u)<\alpha$, in contradiction to Proposition 1 since $\delta(u)=\delta(w) \geq \alpha$. Thus $k \geq \ell$, from which follows $v \in \mathcal{L}$.

Corollary 1 Let $\alpha \in(0,1]$ and $w \in \mathcal{L}_{\text {fin }}$ with $\delta(w) \geq \alpha$. Then $v=$ lazy-flipext $^{\omega}(w, \alpha)$ is an infinite prefix normal word and $\delta(v)=\alpha$.

Proof. That $v$ is prefix normal follows from Lemma 1 and from Lemma 2, which also implies that $\delta(v) \geq \alpha$. However, if $\delta(v)>\alpha$ was true, then for a suitably long prefix $i$, we would get a contradition to the definition of the lazy-flipext operation.

Fix $w \in \mathcal{L}_{\text {fin }}$. The next proposition states that the lazy-flipext operation with $\alpha=\delta(w)$, applied to $w$, generates a prefix normal word that has the minimum number of 1 s among all prefix normal words with prefix $w$ and minimum density $\delta(w)$.

Proposition 3 Let $w \in \mathcal{L}_{\text {fin }}, \alpha=\delta(w), v=$ lazy-flipext $^{\omega}(w, \alpha)$, and $z \in \mathcal{L}_{\text {inf }}$ such that $\operatorname{pref}_{z}(|w|)=w$ and $\delta(z) \geq \delta(w)$. Then for all $i=1,2, \ldots$, we have $P_{v}(i) \leq P_{z}(i)$. 
Proof. We argue by contradiction. Let $i$ be the smallest integer such that $P_{v}(i)<P_{z}(i)$. Clearly $i>|w|$ and, by the minimality assumption, we have $P_{v}(i-1)=P_{z}(i-1)$ and $v_{i}=0, z_{i}=1$. Let $u=\operatorname{pref}_{v}(i-1)$. Since $i>|w|$ and $v_{i}=1$, therefore $u 1=\operatorname{lazy-flipext}\left(u^{\prime}, \alpha\right)$ for some $u^{\prime}$, and thus, by definition of lazy-flipext, $P_{u 0}(i) / i<\alpha$. But $u 0=\operatorname{pref}_{i}(z)$, so we have

$$
\delta(z) \leq D_{z}(i)=\frac{P_{z}(i)}{i}=\frac{P_{u 0}(i)}{i}<\delta(w),
$$

in contradiction to the density of $z$.

Theorem 1 Let $w \in \mathcal{L}_{\text {fin }}$ with $\alpha=\delta(w) \in(0,1]$, and let $z \in \mathcal{L}_{\text {inf }}$ such that $\operatorname{pref}_{z}(|w|)=w$ and $\delta(z) \geq \alpha$. Let $u=$ flipext $^{\omega}(w)$ and $v=\operatorname{lazy}-f l i p e x t^{\omega}(w, \alpha)$. Then $v \leq_{\text {lex }} z \leq_{\text {lex }} u$.

Proof. Follows from Prop. 2 and Prop. 3.

Note that if $\operatorname{pref}_{z}(|w|)=w$, then $\delta(z) \geq \delta(w)$ implies that, in fact, $\delta(z)=\delta(w)$ holds, since $z$ is an extension of $w$. Theorem 1 states then that all prefix normal extensions of $w$ with the same minimum density as $w$ lie lexicographically between the lazy-flipext- and the flipext-extensions of $w$. However, not all extensions of $w$ between these two words are prefix normal, as we can see in the next example.

Example 3 Let $w=1101101100100010000001$, with $\alpha=\delta(w)=8 / 21$, then

$$
\begin{aligned}
& v=\text { lazy-flipext }^{(8)}(w, \alpha)=w 01001010010010100100 \\
& u=\text { flipext }^{(8)}(w)=w 101101100100010000001
\end{aligned}
$$

Let $p=w 100111010100000100001$ and $q=w 101101010100001000001$, we have that for all $1 \leq i \leq 42, P_{v}(i) \leq P_{p}(i), P_{q}(i) \leq P_{u}(i)$ and $v \leq_{\operatorname{lex}} p, q \leq_{\operatorname{lex}} u$. Note that $p$ is not prefix normal, while $q$ is prefix normal.

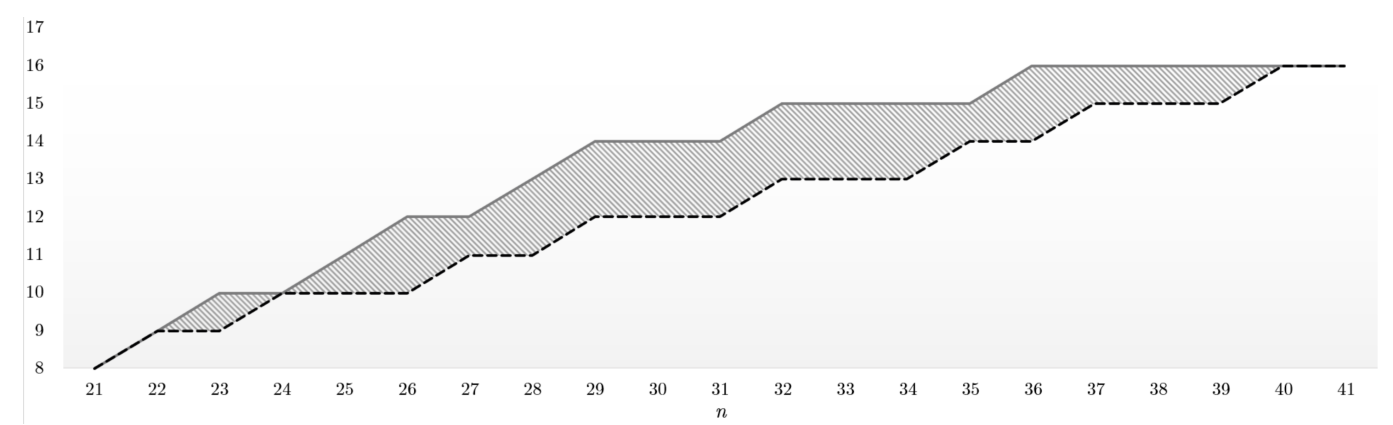

Figure 1: Given $w=1101101100100010000001$ the plot represents the last characters of flipext $^{(8)}(w)$ (solid) and the lazy-flipext ${ }^{(8)}(w, \alpha)$ (dashed). See Example 3. A 1 corresponds to a diagonal segment in direction $\mathrm{NE}$, while a 0 to one in direction SE. On the $x$-axis we have the length of the prefix, and on the $y$-axis, the number of $1 \mathrm{~s}$ minus the number of $0 \mathrm{~s}$ in the prefix. The shaded area contains all prefix normal words with $w$ as prefix and minimum density equal to $\delta(w)$. Note, however, that not all words in that area are prefix normal.

\section{Sturmian words and prefix normal words}

In the previous section, we presented operations that construct infinite prefix normal words by extending finite prefix normal words. In particular, the lazy-flipext operation extends a finite 
binary word with as few 1 s as possible while preserving its minimum density. This is reminiscent of the characterization of Sturmian words in terms of mechanical words and the slope. Led by this analogy, in this section we provide a complete characterization of Sturmian words which are prefix normal. We refer the interested reader to [23, Chapter 2], for a comprehensive treatment of Sturmian words. Here we briefly recall some facts which we will need later.

Definition 6 (Sturmian words) Let $w \in\{0,1\}^{\omega}$. Then $w$ is called Sturmian if it is balanced and aperiodic.

An equivalent definition of Sturmian words is that they are irrational mechanical, a definition we recall next.

Definition 7 (Mechanical words) Given two real numbers $0 \leq \alpha \leq 1$ and $0 \leq \tau<1$, the lower mechanical word $s_{\alpha, \tau}=s_{\alpha, \tau}(1) s_{\alpha, \tau}(2) \cdots$ and the upper mechanical word $s_{\alpha, \tau}^{\prime}=$ $s_{\alpha, \tau}^{\prime}(1) s_{\alpha, \tau}^{\prime}(2) \cdots$ are given by

$$
\begin{aligned}
& s_{\alpha, \tau}(n)=\lfloor\alpha n+\tau\rfloor-\lfloor\alpha(n-1)+\tau\rfloor \\
& s_{\alpha, \tau}^{\prime}(n)=\lceil\alpha n+\tau\rceil-\lceil\alpha(n-1)+\tau\rceil
\end{aligned} \quad(n \geq 1) .
$$

Then $\alpha$ is called the slope and $\tau$ the intercept of $s_{\alpha, \tau}, s_{\alpha, \tau}^{\prime}$. A word $w$ is called mechanical if $w=s_{\alpha, \tau}$ or $w=s_{\alpha, \tau}^{\prime}$ for some $\alpha, \tau$. It is called rational mechanical (resp. irrational mechanical) if $\alpha$ is rational (resp. irrational).

Fact 1 (Some facts about Sturmian words [23]) 1. An infinite binary word is Sturmian if and only if it is irrational mechanical.

2. For $\tau=0$ and irrational $\alpha$, there exists a word $c_{\alpha}$, called the characteristic word with slope $\alpha$, s.t. $s_{\alpha, 0}=0 c_{\alpha}$ and $s_{\alpha, 0}^{\prime}=1 c_{\alpha}$. This word $c_{\alpha}$ is a Sturmian word itself, with both slope and intercept $\alpha$.

3. For two Sturmian words $w$ and $v$ with the same slope, $F c t(w)=F c t(v)$.

We now show that the word lazy-flipext ${ }^{\omega}(1, \alpha)$ coincides with the upper mechanical word $s_{\alpha, 0}^{\prime}$. This also implies that $s_{\alpha, 0}^{\prime}$ is prefix normal, as noted in the subsequent corollary.

Lemma 3 Fix $\alpha \in(0,1]$ and let $v=$ lazy-flipext ${ }^{\omega}(1, \alpha)$. Let $s=s_{\alpha, 0}^{\prime}$ be the upper mechanical word of slope $\alpha$ and intercept 0 . Then $v=s$.

Proof. Let $s_{i}$ and $v_{i}$ denote the $i$ th character of $s$ and $v$ respectively. We argue by induction on $i$ that $v_{i}=s_{i}$. The claim is true for $i=1$ since, directly from the definitions we have $v_{1}=1=s_{1}$. Let $n>1$ and assume that for each $i<n$ we have $v_{i}=s_{i}$. For the induction step we argue according to the character $s_{n}$.

(i) If $s_{n}=1$, by definition $\lceil n \alpha\rceil-\lceil(n-1) \alpha\rceil=1$. Thus, $\lceil(n-1) \alpha\rceil<n \alpha$. Using this inequality and the induction hypothesis together with the definition of $s_{\alpha, 0}^{\prime}$ we have that $\left|v_{1} \cdots v_{n-1}\right|_{1}=$ $\left|s_{1} \cdots s_{n-1}\right|_{1}=\lceil(n-1) \alpha\rceil<\alpha n$. Therefore $\left|v_{1} \cdots v_{n-1} 0\right|_{1}=\left|v_{1} \cdots v_{n-1}\right|_{1}<\alpha n$, which means that $\delta\left(v_{1} \cdots v_{n-1} 0\right)<\alpha$, hence by definition lazy-flipext $\left(v_{1} \cdots v_{n-1}, \alpha\right)=v_{1} \cdots v_{n-1} 1$, i.e., $v_{n}=$ $1=s_{n}$.

(ii) If $s_{n}=0$, by definition $\lceil n \alpha\rceil-\lceil(n-1) \alpha\rceil=0$. Thus, $\lceil(n-1) \alpha\rceil \geq n \alpha$. Using this inequality and the induction hypothesis together with the definition of $s_{\alpha, 0}^{\prime}$ we have that $\left|v_{1} \cdots v_{n-1}\right|_{1}=$ $\left|s_{1} \cdots s_{n-1}\right|_{1}=\lceil(n-1) \alpha\rceil \geq \alpha n$. Therefore $\left|v_{1} \cdots v_{n-1} 0\right|_{1}=\left|v_{1} \cdots v_{n-1}\right|_{1} \geq \alpha n$ which means that $\delta\left(v_{1} \cdots v_{n-1} 0\right) \geq \alpha$, hence by definition lazy-flipext $\left(v_{1} \cdots v_{n-1}, \alpha\right)=v_{1} \cdots v_{n-1} 0 \cdots 01$, i.e., $v_{n}=0=s_{n}$. 
Corollary 2 Let $\alpha \in(0,1]$. Then $s_{\alpha, 0}^{\prime}$ is an infinite prefix normal word and $\delta\left(s_{\alpha, 0}^{\prime}\right)=\alpha$.

The following theorem fully characterizes those Sturmian words which are prefix normal.

Theorem 2 A Sturmian word $s$ of slope $\alpha$ is prefix normal if and only if $s=1 c_{\alpha}$, where $c_{\alpha}$ is the characteristic Sturmian word with slope $\alpha$.

Proof. By definition, $\alpha$ is irrational. Let $s=s_{\alpha, 0}^{\prime}$. Then $s$ is Sturmian and prefix normal by Corollary 2, Let $t$ be a Sturmian word with the same slope $\alpha$ which is also prefix normal. By Fact 1, $s$ and $t$ have the same factors.

Assume, by contradiction, that $s \neq t$, hence there exists $i \geq 1$ such that $\left|s_{1} \cdots s_{i}\right|_{1} \neq$ $\left|t_{1} \cdots t_{i}\right|_{1}$. Assume, without loss of generality (since we can, if necessary, swap $s$ and $t$ in the following argument), that $\left|s_{1} \cdots s_{i}\right|_{1}>\left|t_{1} \cdots t_{i}\right|_{1}$. Then, since $s_{1} \cdots s_{i}$ is also a factor of $t$, there is a $j \geq 1$ such that $t_{j+1} \cdots t_{j+i}=s_{1} \cdots s_{i}$, hence $\left|t_{j+1} \cdots t_{j+i}\right|_{1}>\left|t_{1} \cdots t_{i}\right|_{1}$ contradicting the assumption that $t$ is prefix normal.

\section{Prefix normal words, prefix normal forms, and abelian complexity}

Given an infinite word $w$, the abelian complexity function of $w$, denoted $\psi_{w}$, is given by $\psi_{w}(n)=$ $|\{p v(u)|u \in F c t(w)| u \mid,=n\}|$, the number of Parikh vectors of $n$-length factors of $w$. A word $w$ is said to have bounded abelian complexity if there exists a $c$ s.t. for all $n, \psi_{w}(n) \leq c$. Note that a binary word is $c$-balanced if and only if its abelian complexity is bounded by $c+1$. We denote the set of Parikh vectors of factors of a word $w$ by $\Pi(w)=\{p v(u) \mid u \in F c t(w)\}$. Thus, $\psi_{w}(n)=|\Pi(w) \cap\{(x, y) \mid x+y=n\}|$. In this section, we study the connection between prefix normal words and abelian complexity.

\subsection{Balanced and $c$-balanced words.}

Based on the examples in the introduction, one could conclude that any word with bounded abelian complexity can be turned into a prefix normal word by prepending a fixed number of $1 \mathrm{~s}$. However, consider the word $w=01^{\omega}$, which is balanced, i.e. its abelian complexity function is bounded by 2 . It is easy to see that $1^{k} w \notin \mathcal{L}$ for every $k \in \mathbb{N}$.

Sturmian words are precisely the words which are aperiodic and whose abelian complexity is constant 2 [27. For Sturmian words, it is always possible to prepend a finite number of 1s to get a prefix normal word, as we will see next. Recall that for a Sturmian word $w$, at least one of $0 w$ and $1 w$ is Sturmian, with both being Sturmian if and only if $w$ is characteristic [23.

Lemma 4 Let $w$ be a Sturmian word with slope $\alpha$. Then

1. $1 w \in \mathcal{L}$ if and only if $0 w$ is Sturmian,

2. if $0 w$ is not Sturmian, then $1^{n} w \in \mathcal{L}$ for $n=\lceil 1 /(1-\alpha)\rceil$.

Proof. 1. Let $0 w$ be Sturmian and let $u$ be some factor of $1 w$. If $u$ is a prefix of $1 w$, there is nothing to show, therefore let $u \in F c t(w)$, with $|u|=n$ and $|u|_{1}=k$. Since $0 w$ is Sturmian, we have that the prefix of $0 w$ of length $n$ has at least $k-11$ s, thus $P_{1 w}(n) \geq k=|u|_{1}$, as desired. Conversely, if $0 w$ is not Sturmian, this means that it is not balanced, therefore there exists a factor $u$ of $w$ s.t. $\left.|| u\right|_{1}-\left|0 w_{1} \cdots w_{n-1}\right|_{1} \mid \geq 2$, where $|u|=n$. Since $w$ is Sturmian, we have that $\left.|| w_{1} \cdots w_{n-1}\right|_{1}-\left|u_{1} \cdots u_{n-1}\right|_{1} \mid \leq 1$ and $\left.|| w_{1} \cdots w_{n-1}\right|_{1}-\left|u_{2} \cdots u_{n}\right|_{1} \mid \leq 1$. Let $\left|w_{1} \cdots w_{n-1}\right|_{1}=k$, 
then this implies, by a case-by-case consideration, that $\left|u_{1} \cdots u_{n-1}\right|_{1}=\left|u_{2} \cdots u_{n}\right|_{1}=k+1$, and thus $\left|1 w_{1} \cdots w_{n-1}\right|_{1}=k+1<k+2=|u|_{1}$, showing that $1 w$ is not prefix normal.

2. First note that a Sturmian word of slope $\alpha$ cannot have a run of 1 s of length $\lceil 1 /(1-\alpha)\rceil$. To see this, it is enough to consider the upper mechanical word of slope $\alpha$ and intercept 0 (since all the other words with the same slope have the same set of factors). Let us write $s=s_{\alpha, 0}=s_{1} s_{2} \cdots$

Now $s$ has a run of $n 1 \mathrm{~s}$ if and only if there exists an $i \geq 0$ such that $s_{i+1}=s_{i+2}=\cdots=$ $s_{i+n}=1$. By the definition of mechanical words, we have that the last condition is equivalent to

$$
\lceil\alpha(i+n)\rceil-\lceil\alpha i\rceil=n \text {. }
$$

On the other hand, if $n \geq \frac{1}{1-\alpha}$, i.e., $\alpha \leq \frac{n-1}{n}$ we have that the sum of the character $\sum_{j=1}^{n} s_{i+j}$ satisfies

$$
\begin{aligned}
\sum_{j=1}^{n} s_{i+j} & =\lceil\alpha(i+n)\rceil-\lceil\alpha i\rceil \leq\lceil\alpha i\rceil+\lceil\alpha n\rceil-\lceil\alpha i\rceil \\
& =\lceil\alpha n\rceil<\alpha n+1 \leq \frac{n-1}{n} \times n+1=n .
\end{aligned}
$$

i.e., strictly smaller than $n$, i.e., we have a contradiction $s_{i+1} \cdots s_{i+n} \neq 1^{n}$.

Now fix $n=\lceil 1 /(1-\alpha)\rceil$ and let $w^{\prime}=1^{n} w$. Let $u \in F c t(w)$. Since, as shown above, $1^{n}$ is not a factor, if $|u| \leq n$, there is nothing to show. So let $|u|=n+m$. Then $\left|u_{1} \cdots u_{n}\right|_{1} \leq n-1$, and since $w$ is balanced, we have that $\left|w_{1} \cdots w_{m}\right|_{1} \geq\left|u_{n+1} \cdots u_{n+m}\right|_{1}-1$, yielding that $P_{w^{\prime}}(n+m) \geq$ $n+\left|u_{n+1} \cdots u_{n+m}\right|_{1}-1 \geq|u|_{1}$.

Lemma 5 Let $w$ be a c-balanced word. If there exists a positive integer $n$ s.t. $1^{n} \notin F c t(w)$, then the word $z=1^{n c} w$ is prefix normal.

Proof. We are going to show that every factor $u$ of $z$ satisfies the prefix normal condition $|u|_{1} \leq$ $P_{z}(|u|)$. It is not hard to see that we can limit ourselves to only considering factors $u$ such that $u$ does not overlap with the prefix of $z$ of the same length.

If $|u| \leq n c$ then $|u|_{1} \leq|u|=P_{z}(|u|)$. Assume now that $u=u^{\prime} u^{\prime \prime}$ with $\left|u^{\prime}\right|=n c$ and $\left|u^{\prime \prime}\right|>0$. Since $u^{\prime}$ is a factor of $w$ of size $n c$ the condition that $w$ does not contain a factor $1^{n}$ implies that $u^{\prime}$ contains at least $c 0$ s, i.e., $\left|u^{\prime}\right|_{1} \leq\left|u^{\prime}\right|-c$. Moreover, since $w$ is $c$-balanced, we have that $\left|u^{\prime \prime}\right|_{1} \leq P_{w}\left(\left|u^{\prime \prime}\right|\right)+c$. Therefore, observing that $\operatorname{pref}_{z}(|u|)=\operatorname{pref}_{z}\left(\left|u^{\prime}\right|+\left|u^{\prime \prime}\right|\right)=1^{n c} \operatorname{pref}_{w}\left(\left|u^{\prime \prime}\right|\right)$ we have that $P_{z}(|u|)=n c+P_{w}\left(\left|u^{\prime \prime}\right|\right) \geq\left|u^{\prime}\right|_{1}+\left|u^{\prime \prime}\right|_{1}=|u|_{1}$.

In particular, Lemma 5 implies that any c-balanced word with infinitely many 0s can be turned into a prefix normal word by prepending a finite number of $1 \mathrm{~s}$, since such a word cannot have arbitrarily long runs of $1 \mathrm{~s}$. Note, however, that the number of 1 s to prepend from Lemma 5 is not tight, as can be seen e.g. from the Thue-Morse word $\mathbf{t}$ : the longest run of $1 \mathrm{~s}$ in $\mathbf{t}$ is 2 and $\mathbf{t}$ is 2-balanced, but $11 \mathfrak{t}$ is prefix normal, as will be shown in the next section (Lemma 8).

\subsection{Prefix normal forms and abelian complexity.}

Recall that for a word $w, F_{w}^{a}(i)$ is the maximum number of $a$ 's in a factor of $w$ of length $i$, for $a \in\{0,1\}$.

Definition 8 (Prefix normal forms) Let $w \in\{0,1\}^{\omega}$. Define the words $w^{\prime}$ and $w^{\prime \prime}$ by setting, for $n \geq 1, w_{n}^{\prime}=F_{w}^{1}(n)-F_{w}^{1}(n-1)$ and $w_{n}^{\prime \prime}=\overline{F_{w}^{0}(n)-F_{w}^{0}(n-1)}$. We refer to $w^{\prime}$ as the prefix normal form of $w$ w.r.t. 1 and to $w^{\prime \prime}$ as the prefix normal form of $w$ w.r.t. 0, denoted $\operatorname{PNF}_{1}(w)$ resp. $\mathrm{PNF}_{0}(w)$. 


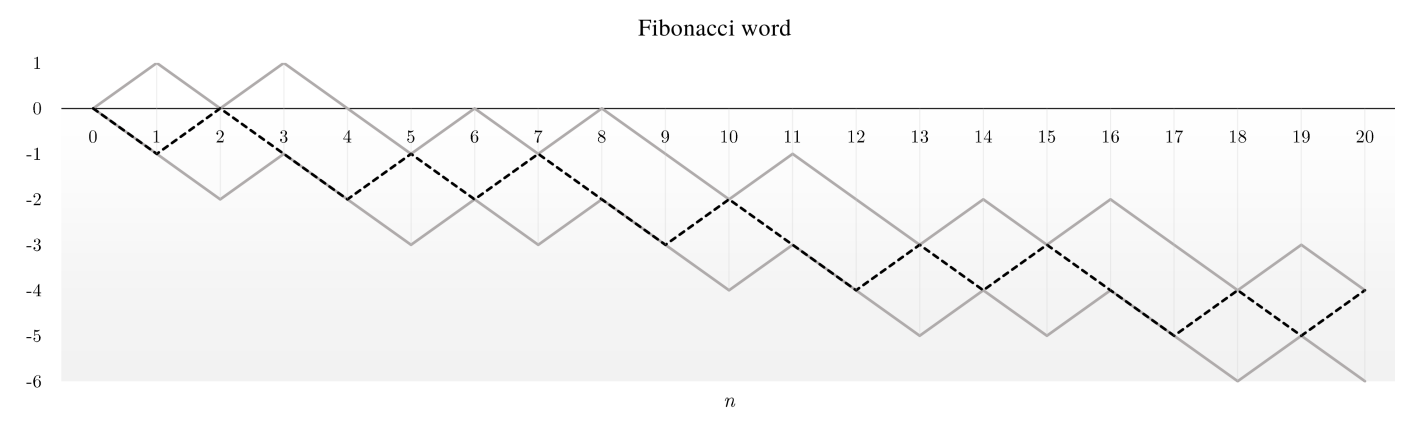

Figure 2: The Fibonacci word (dashed) and its prefix normal forms (solid).

In other words, $\mathrm{PNF}_{1}(w)$ is the sequence of first differences of the maximum-1s function $F_{w}^{1}$ of $w$. Similarly, $\mathrm{PNF}_{0}(w)$ can be obtained by complementing the sequence of first differences of the maximum-0s function $F_{w}^{0}$ of $w$. Note that for all $n$ and $a \in\{0,1\}$, either $F_{w}^{a}(n+1)=F_{w}^{a}(n)$ or $F_{w}^{a}(n+1)=F_{w}^{a}(n)+1$, and therefore $w^{\prime}$ and $w^{\prime \prime}$ are words over the alphabet $\{0,1\}$. In particular, by construction, the two prefix normal words allow us to recover the maximum- $1 \mathrm{~s}$ and minimum-1s functions of $w$ :

Observation 1 Let $w$ be an infinite binary word and $w^{\prime}=\operatorname{PNF}_{1}(w), w^{\prime \prime}=\operatorname{PNF}_{0}(w)$. Then $P_{w^{\prime}}(n)=F_{w}^{1}(n)$ and $P_{w^{\prime \prime}}(n)=n-F_{w}^{0}(n)=f_{w}^{1}(n)$.

Lemma 6 Let $w \in\{0,1\}^{\omega}$. Then $\operatorname{PNF}_{1}(w)$ is the unique 1-prefix normal word $w^{\prime}$ s.t. for all $i \in \mathbb{N}, F_{w^{\prime}}^{1}(i)=F_{w}^{1}(i)$. Similarly, $\operatorname{PNF}_{0}(w)$ is the unique 0-prefix normal word $w^{\prime \prime}$ s.t. for all $i \in \mathbb{N}, F_{w^{\prime \prime}}^{0}(i)=F_{w}^{0}(i)$.

Proof. Let $w^{\prime}=\operatorname{PNF}_{1}(w)$ and $w^{\prime \prime}=\operatorname{PNF}_{0}(w)$. First note that, by construction, for all $i \in \mathbb{N}$, $F_{w^{\prime}}^{1}(i)=F_{w}^{1}(i)$ and $F_{w^{\prime \prime}}^{0}(i)=F_{w}^{0}(i)$. It is easy to see that $w^{\prime}$ is 1-prefix normal and $w^{\prime \prime}$ is 0 -prefix normal. For uniqueness, note that for $a \in\{0,1\}$ and an $a$-prefix normal word $v$, we have $\mathrm{PNF}_{a}(v)=v$.

Example 4 The two prefix normal forms and the maximum-1s and maximum-0s functions of the Fibonacci word $\mathfrak{f}=01001010010010100101 \cdots$ are given in Table 1 .

\begin{tabular}{c|cccccccccccccccccccc}
\hline $\mathrm{n}$ & 1 & 2 & 3 & 4 & 5 & 6 & 7 & 8 & 9 & 10 & 11 & 12 & 13 & 14 & 15 & 16 & 17 & 18 & 19 & 20 \\
\hline$F_{\mathfrak{f}}^{0}(n)$ & 1 & 2 & 2 & 3 & 4 & 4 & 5 & 5 & 6 & 7 & 7 & 8 & 9 & 9 & 10 & 10 & 11 & 12 & 12 & 13 \\
$F_{\mathfrak{f}}^{1}(n)$ & 1 & 1 & 2 & 2 & 2 & 3 & 3 & 4 & 4 & 4 & 5 & 5 & 5 & 6 & 6 & 7 & 7 & 7 & 8 & 8 \\
\hline $\mathrm{PNF}_{0}(\mathfrak{f})$ & 0 & 0 & 1 & 0 & 0 & 1 & 0 & 1 & 0 & 0 & 1 & 0 & 0 & 1 & 0 & 1 & 0 & 0 & 1 & 0 \\
$\mathrm{PNF}_{1}(\mathfrak{f})$ & 1 & 0 & 1 & 0 & 0 & 1 & 0 & 1 & 0 & 0 & 1 & 0 & 0 & 1 & 0 & 1 & 0 & 0 & 1 & 0 \\
\hline
\end{tabular}

Table 1: The maximum number of 0 s and $1 \mathrm{~s}\left(F_{\mathfrak{f}}^{0}(n)\right.$ and $F_{\mathfrak{f}}^{1}(n)$ resp. $)$ for all $n=1, \ldots, 20$ of the Fibonacci word $\mathfrak{f}$, and the prefix normal forms of $\mathfrak{f}$.

Now we can connect the prefix normal forms of $w$ to the abelian complexity of $w$ in the following way. Given $w^{\prime}=\operatorname{PNF}_{1}(w)$ and $w^{\prime \prime}=\operatorname{PNF}_{0}(w)$, the number of Parikh vectors of $k$ length factors is precisely 1 more than the difference in 1s in the prefix of length $k$ of $w^{\prime}$ and of $w^{\prime \prime}$. For example, Fig. 2 shows the prefix normal forms of the Fibonacci word. The vertical line at 5 cuts through points $(5,-1)$ and $(5,-3)$ : the first component stands for the length of the 


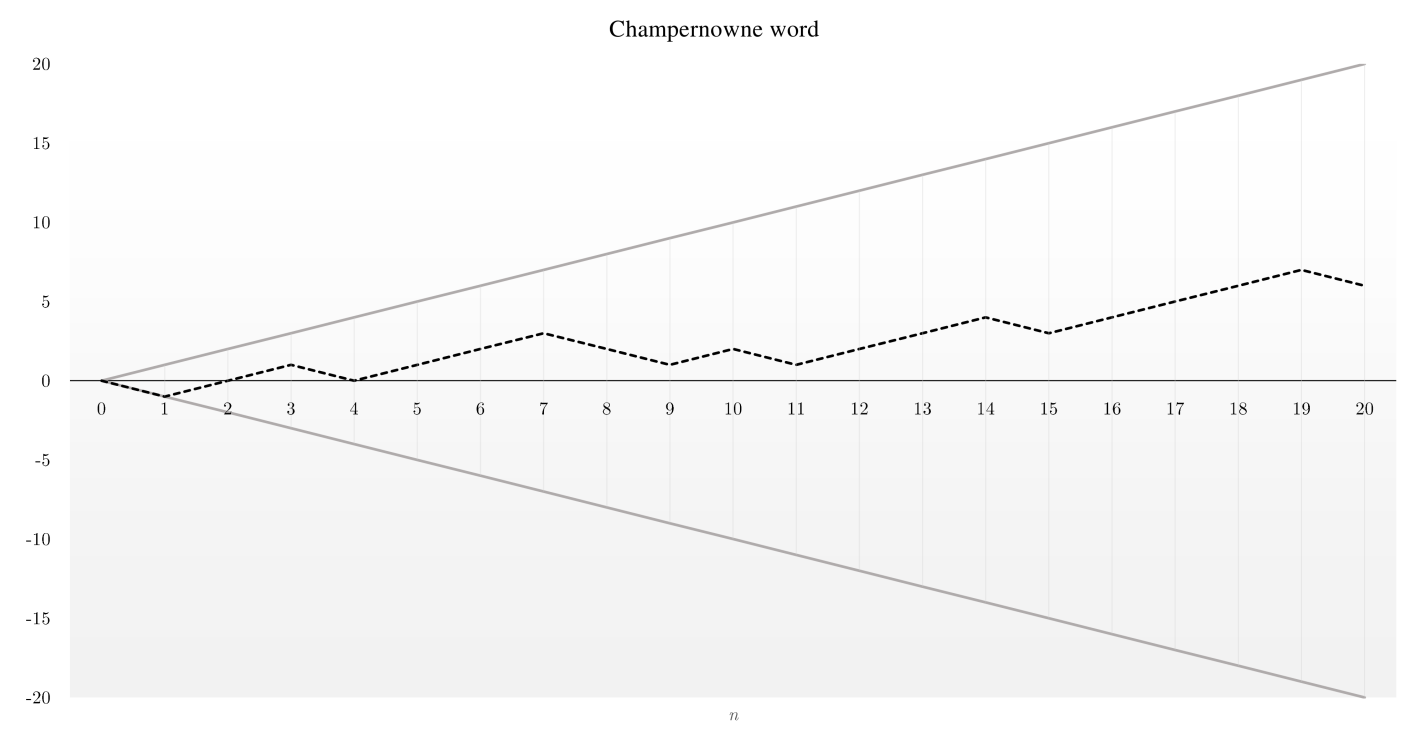

Figure 3: The Champernowne word (dashed) and its prefix normal forms (solid).

string, the second for the difference between the number of $0 \mathrm{~s}$ and the number of $1 \mathrm{~s}$, therefore indicating Parikh vectors $(2,3)$ and $(1,4)$.

The Fibonacci word, being a Sturmian word, has constant abelian complexity 2. An example of a word with unbounded abelian complexity is the Champernowne word, whose prefix normal forms are $1^{\omega}$ resp. $0^{\omega}$. (Fig. 33).

Theorem 3 Let $w, v \in\{0,1\}^{\omega}$.

1. $\psi_{w}(n)=P_{w^{\prime}}(n)-P_{w^{\prime \prime}}(n)+1$, where $w^{\prime}=\operatorname{PNF}_{1}(w)$ and $w^{\prime \prime}=\operatorname{PNF}_{0}(w)$.

2. $\Pi(w)=\Pi(v)$ if and only if $\operatorname{PNF}_{0}(w)=\operatorname{PNF}_{0}(v)$ and $\operatorname{PNF}_{1}(w)=\operatorname{PNF}_{1}(v)$.

Proof. 1. Fix an integer $n \geq 1$. By definition, we have that for every factor $u$ of $w$ of length $n$ we have $n-F_{w}^{0}(n) \leq|u|_{1} \leq F_{w}^{1}(n)$. Therefore $\psi_{w}(n) \leq F_{w}^{1}(n)-\left(n-F_{w}^{0}(n)\right)+1$.

Conversely, since $w$ contains a factor $u^{\prime}$ of length $n$ with $F_{w}^{1}(n)$ many 1 s and a factor $u^{\prime \prime}$ of length $n$ with $n-F_{w}^{0}(n)$ many $1 \mathrm{~s}$, if we scan $w$ between an occurrence of $u^{\prime}$ and an occurrence of $u^{\prime \prime}$, for each $x \in\left\{\left|u^{\prime \prime}\right|_{1}, \ldots,\left|u^{\prime}\right|_{1}\right\}$ there must be a factor $u^{\prime \prime \prime}$ of size $n$ such that $\left|u^{\prime \prime \prime}\right|_{1}=x$. Therefore $\psi_{w}(n) \geq F_{w}^{1}(n)-\left(n-F_{w}^{0}(n)\right)+1$. We can conclude that $\psi_{w}(n)=F_{w}^{1}(n)-(n-$ $\left.F_{w}^{0}(n)\right)+1$. The desired result then follows by observing that $n-F_{w}^{0}(n)=n-\left|\operatorname{pref}_{\mathrm{PNF}_{0}(w)}(n)\right|_{0}=$ $P_{\mathrm{PNF}_{0}(w)}(n)$ and $F_{w}^{1}(n)=P_{\mathrm{PNF}_{1}(w)}(n)$.

2. Follows directly from Observation 1 .

Theorem 3 implies that if we know the prefix normal forms of a word, then we can compute its abelian complexity. Conversely, the abelian complexity is the width of the area enclosed by the two words $\mathrm{PNF}_{1}(w)$ and $\mathrm{PNF}_{0}(w)$. In general, this fact alone does not give us the PNFs; but if we know more about the word itself, then we may be able to compute the prefix normal forms, as we will see in the case of the paperfolding word.

We will now give two examples of the close connection between abelian complexity and prefix normal forms, using some recent results about the abelian complexity of infinite words. 


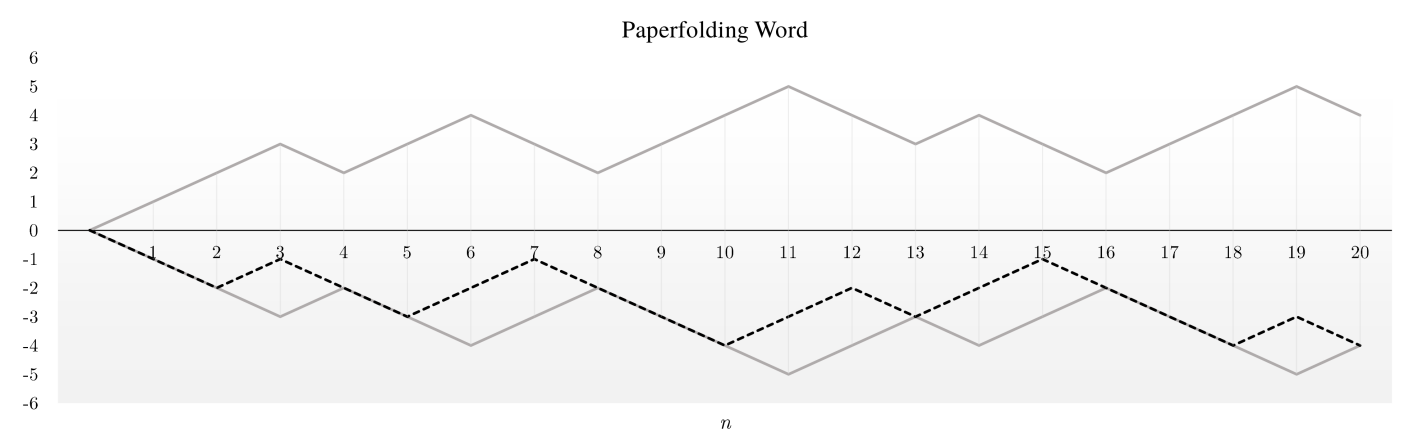

Figure 4: The paperfolding word (dashed) and its prefix normal forms (solid).

\subsubsection{The paperfolding word}

The first few characters of the ordinary paperfolding word are given by

$$
\mathfrak{p}=0010011000110110001001110011011 \cdots
$$

The paperfolding word was originally introduced in [17]. One definition is given by: $\mathfrak{p}_{n}=0$ if $n^{\prime} \equiv 1 \bmod 4$ and $\mathfrak{p}_{n}=1$ if $n^{\prime} \equiv 3 \bmod 4$, where $n^{\prime}$ is the unique odd integer such that $n=n^{\prime} 2^{k}$ for some $k$ [24]. The abelian complexity function of the paperfolding word was fully determined in 24], giving the following initial values for $\psi_{\mathfrak{p}}(n)$, for $n \geq 1: 2,3,4,3,4,5,4,3,4,5,6,5,4,5,4,3,4,5,6,5$, and a recursive formula for the computation of all values. The authors note that for the paperfolding word, it holds that if $u \in F \operatorname{ct}(\mathfrak{p})$, then also $\overline{u^{\mathrm{rev}}} \in \operatorname{Fct}(\mathfrak{p})$. This implies

$$
F_{\mathfrak{p}}^{1}(n)=F_{\mathfrak{p}}^{0}(n) \text { for all } n \text {, and thus } \mathrm{PNF}_{0}(\mathfrak{p})=\overline{\mathrm{PNF}_{1}(\mathfrak{p})}
$$

Moreover, from Thm. 3 we get that $F_{\mathfrak{p}}^{1}(n)=P_{\mathrm{PNF}_{1}(\mathfrak{p})}(n)=\left(\psi_{\mathfrak{p}}(n)+n-1\right) / 2$, and thus we can determine the prefix normal forms of $\mathfrak{p}$, see Fig. 4.

This same argument holds in general as long as the word has the symmetric property similar to the paperfolding word. Therefore, we have proved the following lemma.

Lemma 7 Let $w \in\{0,1\}^{\omega}$. If for all $u \in F c t(w)$, it holds that $\bar{u} \in F c t(w)$ or $\overline{u^{\mathrm{rev}}} \in F c t(w)$, then $F_{w}^{1}(n)=F_{w}^{0}(n)$ for all $n, \mathrm{PNF}_{0}(w)=\overline{\operatorname{PNF}_{1}(w)}$, and $F_{w}^{1}(n)=\left(\psi_{w}(n)+n-1\right) / 2$.

\subsubsection{Morphic images under the Thue-Morse morphism}

The Thue-Morse word beginning with 0 , which we denote by $\mathbf{t}$, is one of the two fix points of the Thue-Morse morphism $\mu_{\mathrm{TM}}$, where $\mu_{\mathrm{TM}}(0)=01$ and $\mu_{\mathrm{TM}}(1)=10$ :

$$
\mathfrak{t}=\mu_{\mathrm{TM}}^{\omega}(0)=01101001100101101001011001101001 \cdots
$$

The word $\mathbf{t}$ has abelian complexity function $\psi_{\mathbf{t}}(n)=2$ for $n$ odd and $\psi_{\mathbf{t}}(n)=3$ for $n>1$ even [27. Since $\mathbf{t}$ fulfils the condition that $u \in F c t(\mathbf{t})$ implies $\bar{u} \in F \operatorname{ct}(\mathbf{t})$, we can apply Lemma 7 , and compute the prefix normal forms of $\mathfrak{t}$ as $\mathrm{PNF}_{1}(\mathbf{t})=1(10)^{\omega}$ and $\mathrm{PNF}_{0}(\mathbf{t})=0(01)^{\omega}$, see Fig. 5 .

For the proof of the abelian complexity of $\mathfrak{t}$ in [27], the Parikh vectors were computed for each length, so we do not really need Lemma 7 but could have obtained the prefix normal forms directly. Moreover, a much more general result was given in [27]: 


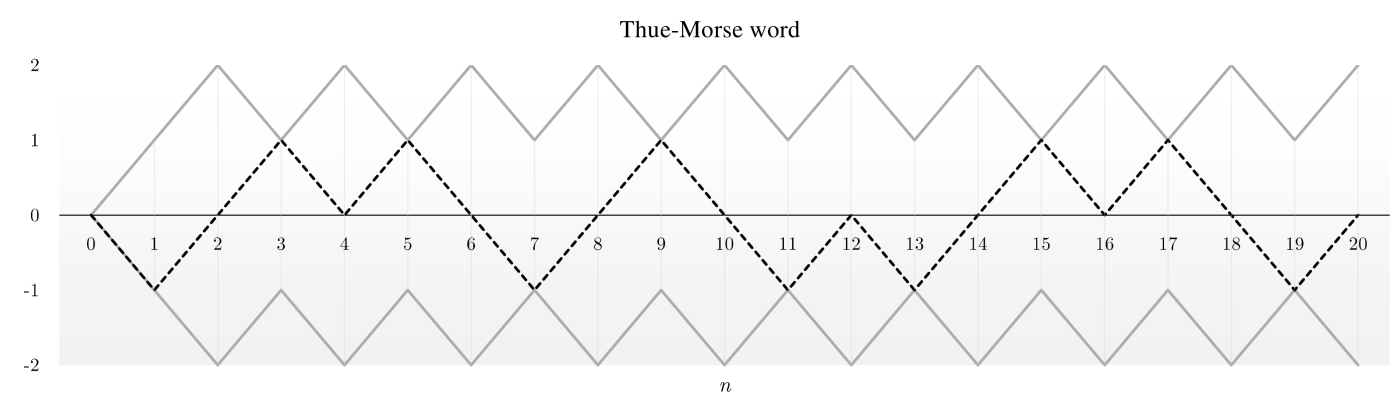

Figure 5: The Thue-Morse word (dashed) and its prefix normal forms (solid).

Theorem $4([\mathbf{2 7}])$ Let $w$ be an aperiodic infinite binary word. Then $\psi_{w}=\psi_{\mathbf{t}}$ if and only if $w=\mu_{T M}\left(w^{\prime}\right), w=0 \mu_{T M}\left(w^{\prime}\right)$, or $w=1 \mu_{T M}\left(w^{\prime}\right)$, for some word $w^{\prime}$.

The abelian complexity function does not in general determine the prefix normal forms, as can be seen on the example of Sturmian words, which all have the same abelian complexity function but different prefix normal forms. However, $\psi_{\mathfrak{t}}$ does, due to its values $\psi_{\mathfrak{t}}(n)=2$ for $n$ odd and $\psi_{\mathfrak{t}}(n)=3$ for $n$ even, and to the fact that both $F_{\mathfrak{t}}^{1}$ and $F_{\mathbf{t}}^{0}$ have difference function with values from $\{0,1\}$ : notice that the only pair of such functions with width 2 resp. 3 are the PNFs of $\boldsymbol{t}$. Therefore, we can deduce the following from Theorem 4

Corollary 3 For an aperiodic infinite binary word $w, \operatorname{PNF}_{1}(w)=1(10)^{\omega}$ and $\mathrm{PNF}_{0}=0(01)^{\omega}$ if and only if $w=\mu_{T M}\left(w^{\prime}\right), w=0 \mu_{T M}\left(w^{\prime}\right)$, or $w=1 \mu_{T M}\left(w^{\prime}\right)$, for some word $w^{\prime}$.

To conclude this section, we return to the question of how many 1s need to be prepended to make the Thue-Morse word prefix normal.

Lemma 8 We have $11 \mathfrak{t} \in \mathcal{L}$. Moreover, this is minimal since $1 \mathfrak{t}$ is not prefix normal.

Proof. We will show that for every prefix, the number of $1 \mathrm{~s}$ in the prefix of $11 \mathrm{t}$ is greater than or equal to the the number of $1 \mathrm{~s}$ in the prefix of $\operatorname{PNF}_{1}(\mathfrak{t})$ of the same length. Let $v=\operatorname{PNF}_{1}(\mathfrak{t})$ and $u=11 \mathrm{t}$. It is easy to see that $P_{v}(n)=\left\lfloor\frac{n}{2}\right\rfloor+1$ and

$$
P_{u}(n)= \begin{cases}\frac{n}{2}+1 & \text { if } n \text { is even } \\ \left\lfloor\frac{n}{2}\right\rfloor+2 & \text { if } n \text { is odd and } u_{n}=1 \\ \left\lfloor\frac{n}{2}\right\rfloor+1 & \text { if } n \text { is odd and } u_{n}=0\end{cases}
$$

Thus for all $n \geq 1$ it holds that $P_{u}(n) \geq P_{v}(n)$, implying that $11 \mathfrak{t} \in \mathcal{L}$.

For minimality, note that $1 \mathbf{t}$ is not prefix normal, since 11 is a factor of $\mathbf{t}$.

\subsection{Prefix normal forms of Sturmian words.}

Let $w$ be a Sturmian word. As we saw in Sec. 4 the only 1-prefix normal word in the class of Sturmian words with the same slope $\alpha$ is the upper mechanical word $s_{\alpha, 0}^{\prime}=1 c_{\alpha}$.

Theorem 5 Let $w$ be an irrational mechanical word with slope $\alpha$, i.e. a Sturmian word. Then $\operatorname{PNF}_{1}(w)=1 c_{\alpha}$ and $\operatorname{PNF}_{0}(w)=0 c_{\alpha}$, where $c_{\alpha}$ is the characteristic word of slope $\alpha$. 
Proof. Since the characteristic word $c_{\alpha}$ has the same slope as $w$, we have $F c t(w)=F c t\left(c_{\alpha}\right)$ by Fact 1. The abelian complexity of $w$ is constant 2 [27, thus a factor of length $k$ can have either $F_{w}^{1}(k)$ or $F_{w}^{1}(k)-1$ s. Let us call a factor $u$ of $w$ heavy if $|u|_{1}=F_{w}^{1}(k)$, and light otherwise. We have to show that every prefix of $1 c_{\alpha}$ is heavy; this will imply that $1 c_{\alpha}$ is the prefix normal form of $w$. It is known 23] that the prefixes of the characteristic word are precisely the reverses of its right special factors, where a factor $u$ is called right special if both $u 0$ and $u 1$ are factors. Thus, every prefix $v$ of $1 c_{\alpha}$ has the form $v=1 u^{\text {rev }}$, where both $u 1$ and $u 0$ are factors of $w$, implying that $|v|_{1}=\left|1 u^{\mathrm{rev}}\right|_{1}=|u 1|_{1}=F_{w}^{1}(|u|+1)$, therefore $v=1 u^{\mathrm{rev}}$ is heavy. The fact that $\mathrm{PNF}_{0}(w)=0 c_{\alpha}$ follows analogously.

\subsection{Prefix normal forms of binary uniform morphisms}

In [5] the authors provide an algorithm which computes the abelian complexity of a morphic word that is the fix point of a binary uniform morphism, i.e., a morphism $\mu$ satisfying $|\mu(0)|=|\mu(1)|$. We refer the reader to [5] for the details on this algorithm. In particular, the following theorem is proved in [5]:

Theorem 6 ([5]) Let $w$ be the fix point of a binary uniform morphism $\mu$. Then, for each $n$ the values $\psi_{w}(1), \psi_{w}(2), \ldots, \psi_{w}(n)$, can be computed in $O(n)$ time.

As an intermediate step in the computation of each $\psi_{w}(i)$, the algorithm in [5] provides the minimum number of $0 \mathrm{~s}$ (equivalently, the maximum number of $1 \mathrm{~s}$ ) in every $i$-length factor of $w$. Obviously the same procedure can be used to obtain the minimum number of 1s (equivalently, the maximum number of $0 \mathrm{~s}$ ) in every $i$-length factor of $w$. Therefore, we have the following corollary to the result of [5]:

Corollary 4 Let $w$ be the fix point of a binary uniform morphism $\mu$. For each $n$, the prefix of length $n$ of $\mathrm{PNF}_{1}(w)$ and of $\mathrm{PNF}_{0}(w)$ can be computed in $O(n)$ time.

\section{Prefix normal words and lexicographic order}

In this section, we study the relationship between lexicographic order and prefix normality. Note that for coherence with the rest of the paper, in the definition of Lyndon words, necklaces, and prenecklaces, we use lexicographically greater rather than smaller. Clearly, this is equivalent to the usual definitions up to renaming of characters.

Thus a finite Lyndon word is one which is lexicographically strictly greater than all of its conjugates: $w$ is Lyndon if and only if for all non-empty $u, v$ s.t. $w=u v$, we have $w>_{\text {lex }} v u$. A necklace is a word which is greater than or equal to all its conjugates, and a prenecklace is one which can be extended to become a necklace, i.e. which is the prefix of some necklace [23, 28]. As we saw in the introduction, in the finite case, prefix normality and Lyndon property are orthogonal concepts. However, the set of finite prefix normal words is included in the set of prenecklaces [11].

An infinite word is Lyndon if an infinite number of its prefixes is Lyndon 32. In the infinite case, we have a similar situation as in the finite case. There are words which are both Lyndon and prefix normal: $10^{\omega}, 110(10)^{\omega}$; Lyndon but not prefix normal: $11100(110)^{\omega}$; prefix normal but not Lyndon: $(10)^{\omega}$; and neither of the two: $(01)^{\omega}$.

Next we show that a prefix normal word cannot be lexicographically smaller than any of its suffixes. Let $\operatorname{shift}_{i}(w)=w_{i} w_{i+1} w_{i+2} \cdots$ denote the infinite word $v$ s.t. $w=w_{1} \cdots w_{i-1} v$, i.e. $v$ is the suffix of $w$ starting at position $i$. 
Lemma 9 Let $w \in \mathcal{L}_{\text {inf. }}$ Then $w \geq_{\text {lex }} \operatorname{shift}_{i}(w)$ for all $i \geq 1$.

Proof. Assume that there exists a suffix $v=\operatorname{shift}_{i}(w)$ of $w$ s.t. $v>_{\text {lex }} w$. Then there is an index $j$ with $v_{1} \cdots v_{j-1}=w_{1} \cdots w_{j-1}$ and $v_{j}>w_{j}$, implying $v_{j}=1$ and $w_{j}=0$. But then $\left|w_{i} \cdots w_{i+j-1}\right|_{1}=\left|v_{1} \cdots v_{j}\right|_{1}>\left|w_{1} \cdots w_{j}\right|_{1}$, in contradiction to $w \in \mathcal{L}_{\text {inf }}$.

In the finite case, it is easy to see that a word $w$ is a prenecklace if and only if $w \geq_{\operatorname{lex}} v$ for every suffix $v$ of $w$. This motivates our definition of infinite prenecklaces. The situation is the same as in the finite case: prefix normal words form a proper subset of prenecklaces.

Definition 9 Let $w \in\{0,1\}^{\omega}$. Then $w$ is an infinite prenecklace if for all $i \geq 1, w \geq_{\text {lex }}$ shift $_{i}(w)$. We denote by $\mathcal{P}_{\text {inf }}$ the set of infinite prenecklaces.

Proposition $4 \mathcal{L}_{\text {inf }} \subsetneq \mathcal{P}_{\text {inf }}$.

Proof. The inclusion follows from Lemma 9. An example of a word which is an infinite prenecklace but not prefix normal is $11100(110)^{\omega}$.

There is another interesting relationship between lexicographic order and the prefix normal forms of an infinite word. In [26], two words were associated to an infinite binary word $w$, called $\max (w)$ (resp. $\min (w)$ ), defined as the word whose prefix of length $n$ is the lexicographically greatest (resp. smallest) $n$-length factor of $w$. It is easy to see that these words always exist. The following was shown in [26] ${ }^{3}$

Theorem 7 ([26]) Let $w$ be an infinite binary word. Then

1. $w$ is (rational or irrational) mechanical with its intercept equal to its slope if and only if $0 w \leq_{\operatorname{lex}} \min (w) \leq_{\operatorname{lex}} \max (w) \leq_{\operatorname{lex}} 1 w$, and

2. $w$ is characteristic Sturmian if and only if $\min (w)=0 w$ and $\max (w)=1 w$.

Lemma 10 Let $w \in\{0,1\}^{\omega}$. Then $\operatorname{PNF}_{1}(w) \geq_{\text {lex }} \max (w)$ and $\operatorname{PNF}_{0}(w) \leq_{\text {lex }} \min (w)$.

Proof. Assume otherwise, and let $w^{\prime}=\operatorname{PNF}_{1}(w), v=\max (w)$. If $w^{\prime}<v$, then there is an index $j$ s.t. $w_{1}^{\prime} \cdots w_{j-1}^{\prime}=v_{1} \cdots v_{j-1}$ and $w_{j}^{\prime}=0$ and $v_{j}=1$. This implies that $v_{1} \cdots v_{j}$ has one more 1s than $w_{1}^{\prime} \cdots w_{j}^{\prime}$. But $\left|w_{1}^{\prime} \cdots w_{j}^{\prime}\right|_{1}=F_{w}^{1}(j)$, a contradiction, since $v_{1} \cdots v_{j}$ is a factor of $w$. The second claim follows analogously.

Finally, from Theorems 5 and 7 , we get the following corollary:

Corollary 5 Let $w$ be an infinite binary word. Then $w$ is characteristic Sturmian if and only if $0 w=\operatorname{PNF}_{0}(w)=\min (w)$ and $1 w=\operatorname{PNF}_{1}(w)=\max (w)$.

\footnotetext{
${ }^{3}$ The terminology in 26] differs from ours (we are following 23]). In order to help the reader, here we highlight the differences: $(i)$ a periodic Sturmian in 26 is a rational mechanical word, $(i i)$ a proper Sturmian word in 26 is an irrational mechanical word (i.e., a Sturmian word), and (iii) a standard Sturmian word in [26] is a mechanical word with intercept $\tau=\alpha$ (the slope), thus a proper standard Sturmian word is a characteristic Sturmian word $c_{\alpha}$. Note that all mechanical words in [26] are defined for $n \geq 1$, since the definition of mechanical word is: the lower mechanical word is defined as $s_{\alpha, \tau}(n)=\lfloor\alpha(n+1)+\tau\rfloor-\lfloor\alpha n+\tau\rfloor$ for $n \geq 1$, and analogously for the upper mechanical word. Therefore, an intercept $\tau=0$ in [26] is equivalent to an intercept of $\tau=\alpha$ (the slope) in [23].
} 


\section{On the periodicity and aperiodicity of prefix normal words with respect to minimum density}

In this section, we derive conditions for the periodicity and aperiodicity of prefix normal words with respect to their minimum density. The following result shows that every ultimately periodic infinite prefix normal word has rational minimum density.

Lemma 11 Let $v$ be an infinite ultimately periodic binary word with minimum density $\delta(v)=\alpha$. Then $\alpha \in \mathbb{Q}$.

Proof. Let us write $v=u x^{\omega}$ with $x$ not a suffix of $u$.

For $i=0,1, \ldots,|x|-1$, let $y_{i}$ be the prefix of length $|u|+i$ of $v$, i.e., $y_{i}=u x_{1} x_{2} \cdots x_{i}$. Trivially, if for some $i$ we have that $\delta\left(y_{i}\right) \leq \delta(v)$ the claim directly follows from $y_{i}$ being a finite prefix of $v$.

Let us now assume that for each $i=0,1, \ldots|x|-1$ it holds that $\delta(v)<\delta\left(y_{i}\right)$ and let $i^{*}=\min \left\{i \mid \delta\left(y_{i}\right) \leq \delta\left(y_{j}\right)\right.$ for each $\left.j \neq i\right\}$, hence $\delta(v)<\delta\left(y_{i^{*}}\right)$.

For every $n \geq|u|+|x|$ let $i_{n}=|u|+((n-|u|) \bmod |x|)$ and $k_{n}=\lfloor(n-|u|) /|x|\rfloor$, i.e., $|u| \leq i_{n} \leq|u|+|x|-1$ and $n=i_{n}+k_{n}|x|$.

Then, we have that

$$
D_{v}(n)=\frac{\left|y_{i_{n}}\right|_{1}+k_{n}|x|_{1}}{\left|y_{i_{n}}\right|+k_{n}|x|} \geq \min \left\{\delta\left(y_{i_{n}}\right), \delta(x)\right\} \geq \min \left\{\delta\left(y_{i^{*}}\right), \delta(x)\right\} .
$$

Moreover, we also have that

$$
\lim _{k \rightarrow \infty} D_{v}\left(|u|+i^{*}+k|x|\right)=\lim _{k \rightarrow \infty} \frac{\left|y_{i^{*}}\right|_{1}+k|x|_{1}}{\left|y_{i^{*}}\right|+k|x|}=\delta(x) .
$$

We cannot have $\delta(x) \geq \delta\left(y_{i^{*}}\right)$, since by (1) $\delta\left(y_{i^{*}}\right)$ is a rational lower bound on $D_{v}(n)$ (for each $n \geq 1$ ) which is achieved by $D_{v}\left(|u|+i^{*}\right)$, contradicting the standing hypothesis $\delta(v)<\delta\left(y_{i^{*}}\right)$.

Therefore, we must have $\delta(x)<\delta\left(y_{i^{*}}\right)$, and from (1) we have $D_{v}(n) \geq \delta(x)$ and from (2) we also have that for each $\varepsilon>0$ there exists $k>0$ such that $D_{v}\left(|u|+i^{*}+k|x|\right)<\delta(x)+\varepsilon$. Therefore, $\delta(v)=\inf \left\{D_{v}(n) \mid n \geq 1\right\}=\delta(x)$, which is a rational number, since $x$ is a finite string.

We now show that, while periodicity is characterized by rational density, the converse is not true. It turns out that for every $\alpha \in(0,1)$, both rational and irrational, there exists an aperiodic prefix normal word with minimum density $\alpha$. For irrational $\alpha$, this is an easy corollary from Theorem 2, since the Sturmian word $1 c_{\alpha}$ is prefix normal, and $D(i) \geq \alpha$ for each $i$, therefore, $\delta\left(1 c_{\alpha}\right)=\alpha$. The next lemma shows how to construct an aperiodic prefix normal word with minimum density $\alpha$ for both rational and irrational $\alpha$.

Lemma 12 Fix $\alpha \in(0,1)$, and let $\left(a_{n}\right)_{n \in \mathbb{N}}$ be a strictly decreasing infinite sequence of rational numbers from $(0,1)$ converging to $\alpha$. For each $i=1,2, \ldots$, let the binary word $v^{(i)}$ be defined by

$$
v^{(i)}= \begin{cases}1^{\left\lceil 10 a_{1}\right\rceil} 0^{10-\left\lceil 10 a_{1}\right\rceil} & i=1 \\ \operatorname{pref}_{\text {flipext }{ }^{\omega}\left(v^{(i-1)}\right)}\left(k_{i}\left|v^{(i-1)}\right|\right) 0^{\ell_{i}} & i>1\end{cases}
$$

where $\ell_{i}$ defined by

$$
\ell_{i}= \begin{cases}10-\left\lceil 10 a_{1}\right\rceil & i=1 \\ \left\lfloor k_{i}\left(\frac{\left|v^{(i-1)}\right| 1-a_{i}\left|v^{(i-1)}\right|}{a_{i}}\right)\right\rfloor & i>1,\end{cases}
$$

and $k_{i}$ is the smallest integer greater than one such that $\ell_{i}>\ell_{i-1}$.

Then $v=\lim _{i \rightarrow \infty} v^{(i)}$ is an aperiodic infinite prefix normal word such that $\delta(v)=\alpha$. 
Before proving Lemma 12 , in give an example of the words $v^{(i)}$.

Example 5 We show the first three steps for the construction of an infinite aperiodic word with minimum density $\alpha=1 / 3$ (Lemma 12), using the infinite sequence of rational numbers $a_{i}=i /(3 i-1)$, which tends to $1 / 3$ for $i \rightarrow \infty$. Hence, for $i=1$, we have $a_{1}=1 / 2, \ell_{1}=5$, and $v_{i}=1^{5} 0^{5}$ with minimum density $\delta\left(v_{1}\right)=1 / 2$. At the next step, $a_{2}=2 / 5$, and with the values from the previous iteration we can compute $k_{2}=3$ and $\ell_{2}=7$, hence $v_{2}=1^{5} 0^{5} 1^{5} 0^{5} 1^{5} 0^{5} 0^{7}$, with $\delta\left(v_{2}\right)=15 / 37$. At the third iteration, $a_{3}=3 / 8, k_{3}=3$, and $\ell_{3}=9$, therefore $v_{3}=$ $1^{5} 0^{5} 1^{5} 0^{5} 1^{5} 0^{12} 1^{5} 0^{5} 1^{5} 0^{5} 1^{5} 0^{12} 1^{5} 0^{5} 1^{5} 0^{5} 1^{5} 0^{12} 0^{9}$, and the minimum density is $\delta\left(v_{3}\right)=45 / 120$.

\section{Proof. (of Lemma 12)}

We will first prove the following claim, giving a number of properties of the sequence of words $v^{(i)}$, and then use these to prove that $v$ is aperiodic and $\delta(v)=\alpha$.

Claim. The following properties hold:

1. $\delta\left(v^{(i)}\right) \geq a_{i}$ for each $i \geq 1$;

2. $\iota\left(v^{(i)}\right)=\left|v^{(i)}\right|$ for each $i \geq 1$;

3. $\delta\left(v^{(i)}\right)<\delta\left(v^{(i-1)}\right)$ for each $i \geq 2$;

4. $\left|v^{(i)}\right|_{1}>\left|v^{(i-1)}\right|_{1}$ for each $i \geq 2$;

5. $\delta\left(v^{(i)}\right) \leq a_{i}\left(\frac{k_{i}\left|v^{(i-1)}\right|_{1}}{k_{i}\left|v^{(i-1)}\right|_{1}-a_{i}}\right)$ for each $i \geq 2$.

Proof of the Claim. By direct inspection we have that properties 1 and 2 hold for $v^{(1)}$. We now argue by induction. Fix $i>1$ and let us assume that properties 1 and 2 hold for $v^{(i-1)}$. Then, since $a_{i}<a_{i-1}$ we have

$$
\frac{\left|v^{(i-1)}\right|_{1}}{a_{i}}>\frac{\left|v^{(i-1)}\right|_{1}}{a_{i-1}} \geq\left|v^{(i-1)}\right|
$$

where the last inequality follows from property 1 and 2. Therefore, $\left(\frac{\left|v^{(i-1)}\right|{ }_{1}-a_{i}\left|v^{(i-1)}\right|}{a_{i}}\right)>0$, hence there exists $k_{i}>1$ such that $\left.\mid k_{i}\left(\frac{\left|v^{(i-1)}\right|{ }_{1}-a_{i}\left|v^{(i-1)}\right|}{a_{i}}\right)\right\rfloor>\ell_{i-1}$. In particular, $\ell_{i}$ is well defined.

By property 2 , we have $\iota\left(v^{(i-1)}\right)=\left|v^{(i-1)}\right|$ hence by Proposition 1 , we have $D_{\text {flipext }{ }^{\omega}\left(v^{(i-1)}\right)}\left(k\left|v^{(i-1)}\right|\right)=$ $\delta\left(v^{(i-1)}\right)$ and also $\delta\left(\operatorname{pref}_{\text {flipext }{ }^{\omega}\left(v^{(i-1)}\right)}\left(k_{i}\left|v^{(i-1)}\right|\right)\right)=\delta\left(v^{(i-1)}\right)$.

Moreover, since $\ell_{i}>0$ it is not hard to see from the definition of $v^{(i)}$ that

$$
\delta\left(v^{(i)}\right)=D_{v^{(i)}}\left(\left|v^{(i)}\right|\right)=\frac{k_{i}\left|v^{(i-1)}\right|_{1}}{k_{i}\left|v^{(i-1)}\right|+\ell_{i}}<\delta\left(v^{(i-1)}\right),
$$

which shows that property 3 and property 2 hold for $v^{(i)}$. In addition, because of $k_{i}>1$ and (by Proposition 1), $\left|v^{(i)}\right|_{1}=\left|\operatorname{pref}_{\text {flipext }{ }^{\omega}\left(v^{(i-1)}\right)}\left(k_{i}\left|v^{(i-1)}\right|\right)\right|_{1}=k_{1}\left|v^{(i-1)}\right|_{1}$, it follows that property 4 also holds for $v^{(i)}$.

The definition of $\ell_{i}$, together with the well known property $x-1<\lfloor x\rfloor \leq x$, imply that

$$
\frac{k_{i}}{a_{i}}\left(\left|v^{(i-1)}\right|_{1}-a_{i}\left|v^{(i-1)}\right|\right)-1<\ell_{i} \leq k_{i}\left(\frac{\left|v^{(i-1)}\right|_{1}}{a_{i}}-\left|v^{(i-1)}\right|\right) .
$$


Using the right inequality of (4) in (3), we have $\delta\left(v^{(i)}\right) \geq a_{i}$, showing that property 1 holds for $v^{(i)}$.

In addition, using the left inequality of (4) in (3), we have

$$
\delta\left(v^{(i)}\right) \leq a_{i}\left(\frac{k_{i}\left|v^{(i-1)}\right|_{1}}{k_{i}\left|v^{(i-1)}\right|_{1}-a_{i}}\right)
$$

showing that property 5 holds for $v^{(i)}$. The proof of the claim is complete.

In order to see that $v$ is aperiodic, it is enough to observe that $v \neq 0^{\omega}$ and for each $i \geq 1$ it contains a distinct run of $\ell_{i} 0 \mathrm{~s}$, with $\ell_{i}$ being a strictly increasing sequence.

To show that $\delta(v)=\alpha$, we will prove that $\lim _{i \rightarrow \infty} \delta\left(v^{(i)}\right)=\alpha$. Since $\lim _{i \rightarrow \infty} a_{i}=\alpha$ and for each $i \geq 1, k_{i}>1$ and $\left|v^{(i)}\right|_{1}>\left|v^{(i-1)}\right|{ }_{1}$, we have

$$
\lim _{i \rightarrow \infty} a_{i} \frac{k_{i}\left|v^{(i-1)}\right|_{1}}{k_{i}\left|v^{(i-1)}\right|_{1}-a_{i}}=\lim _{i \rightarrow \infty} a_{i}=\alpha .
$$

Hence, from properties 4 and 5 of the Claim above, we have the desired result, $\lim _{i \rightarrow \infty} \delta\left(v^{(i)}\right)=$ $\lim _{i \rightarrow \infty} a_{i}=\alpha$.

This completes the proof of the lemma.

Summarizing, we have shown the following result.

Theorem 8 For every $\alpha \in(0,1)$ (rational or irrational) there is an infinite aperiodic prefix normal word of minimum density $\alpha$. On the other hand, for every ultimately periodic infinite prefix normal word $w$, the minimum density $\delta(w)$ is a rational number.

\section{Conclusion}

In this paper, we studied infinite prefix normal words. We gave several results of infinite extensions of finite prefix normal words, and we established connections between infinite prefix normal words and other classes of infinite binary words, namely Sturmian words, Lyndon words and max and min words. We provided a complete characterization of prefix normal Sturmian words. Furthermore, we showed that, similar to the finite case, the classes of infinite prefix normal words and Lyndon words are distinct, and that infinite prefix normal words are infinite prenecklaces.

We explored some connections between prefix normal words, prefix normal forms, and abelian complexity. In particular, we showed how to turn balanced and $c$-balanced words without arbitrarily long runs of 1 s into prefix normal words, by prepending a finite number of $1 \mathrm{~s}$. We provided a method to compute the abelian complexity from the prefix normal form of a word, and, for specific cases, we showed how to compute the prefix normal form of a word, given its abelian complexity function. We further applied an existing algorithm to compute the prefix normal form of binary uniform morphisms.

Finally, we gave conditions for the periodicity and the aperiodicity of infinite prefix normal words, according to their minimum density. 


\section{Acknowledgements}

We wish to extend our thanks to the participants of the Workshop on Words and Complexity, which took place in Lyon in February 2018, for exciting discussions and helpful pointers, and to Péter Burcsi, who first got us interested in Sturmian words. We also thank the two anonymous reviewers, whose suggestions helped improve the presentation of our results. MR is funded by the National Science Foundation (NSF) IIS (Grant No. 1618814), IIBR (Grant No. 2029552) and National Institutes of Health (NIH) R01 (Grant No. HG011392).

\section{References}

[1] Peyman Afshani, Ingo van Duijn, Rasmus Killmann, and Jesper Sindahl Nielsen. A lower bound for jumbled indexing. In Proceedings of the 2020 ACM-SIAM Symposium on Discrete Algorithms, (SODA 2020), pages 592-606, 2020.

[2] Amihood Amir, Timothy M. Chan, Moshe Lewenstein, and Noa Lewenstein. On hardness of jumbled indexing. In 41st International Colloquium on Automata, Languages, and Programming (ICALP 2014), volume 8572 of LNCS, pages 114-125, 2014.

[3] Paul Balister and Stefanie Gerke. The asymptotic number of prefix normal words. Theoret. Comput. Sci., 784:75-80, 2019.

[4] Francine Blanchet-Sadri, Nathan Fox, and Narad Rampersad. On the asymptotic abelian complexity of morphic words. Advances in Applied Mathematics, 61:46-84, 2014.

[5] Francine Blanchet-Sadri, Daniel Seita, and David Wise. Computing abelian complexity of binary uniform morphic words. Theor. Comput. Sci., 640:41-51, 2016. doi:10.1016/j. tcs.2016.05.046

[6] Alexandre Blondin Massé, Julien de Carufel, Alain Goupil, Mélodie Lapointe, Émile Nadeau, and Élise Vandomme. Leaf realization problem, caterpillar graphs and prefix normal words. Theoret. Comput. Sci., 732:1-13, 2018.

[7] Péter Burcsi, Ferdinando Cicalese, Gabriele Fici, and Zsuzsanna Lipták. Algorithms for Jumbled Pattern Matching in Strings. International Journal of Foundations of Computer Science, 23:357-374, 2012.

[8] Peter Burcsi, Ferdinando Cicalese, Gabriele Fici, and Zsuzsanna Lipták. On approximate jumbled pattern matching in strings. Theory Comput. Syst., 50(1):35-51, 2012.

[9] Péter Burcsi, Gabriele Fici, Zsuzsanna Lipták, Rajeev Raman, and Joe Sawada. Generating a Gray code for prefix normal words in amortized polylogarithmic time per word. Theor. Comput. Sci., 842:86-99, 2020.

[10] Péter Burcsi, Gabriele Fici, Zsuzsanna Lipták, Frank Ruskey, and Joe Sawada. On combinatorial generation of prefix normal words. In Proc. of the 25th Ann. Symp. on Comb. Pattern Matching (CPM 2014), volume 8486 of LNCS, pages 60-69, 2014.

[11] Péter Burcsi, Gabriele Fici, Zsuzsanna Lipták, Frank Ruskey, and Joe Sawada. On prefix normal words and prefix normal forms. Theoret. Comput. Sci., 659:1-13, 2017.

[12] Julien Cassaigne and Idrissa Kaboré. Abelian complexity and frequencies of letters in infinite words. Int. Journal of Foundations of Computer Science, 27(05):631-649, 2016. 
[13] Timothy M. Chan and Moshe Lewenstein. Clustered integer 3SUM via additive combinatorics. In Proc. of the 47th Ann. ACM on Symp. on Theory of Computing (STOC 2015), pages $31-40,2015$.

[14] Ferdinando Cicalese, Zsuzsanna Lipták, and Massimiliano Rossi. Bubble-flip - A new generation algorithm for prefix normal words. Theoret. Comput. Sci., 743:38-52, 2018.

[15] Ferdinando Cicalese, Zsuzsanna Lipták, and Massimiliano Rossi. On infinite prefix normal words. In Proc. of the 45th International Conference on Current Trends in Theory and Practice of Computer Science (SOFSEM 2019), pages 122-135, 2019.

[16] Luís Felipe I. Cunha, Simone Dantas, Travis Gagie, Roland Wittler, Luis Antonio Brasil Kowada, and Jens Stoye. Faster jumbled indexing for binary RLE strings. In 28th Annual Symposium on Combinatorial Pattern Matching (CPM 2017), pages 19:1-19:9, 2017.

[17] C. Davis and D.E. Knuth. Number representations and dragon curves, I, II. J. Recr. Math., 3:133-149 and 161-181, 1970.

[18] Gabriele Fici and Zsuzsanna Lipták. On prefix normal words. In Proc. of the 15th Intern. Conf. on Developments in Language Theory (DLT 2011), volume 6795 of LNCS, pages 228-238. Springer, 2011.

[19] Pamela Fleischmann, Dirk Nowotka, Mitja Kulczynski, and Danny Bøgsted Poulsen. On collapsing prefix normal words. In Proc. of the 14th International Conference Language and Automata Theory and Applications (LATA 2020), volume 12038 of LNCS, pages 412-424. Springer, 2020.

[20] Travis Gagie, Danny Hermelin, Gad M. Landau, and Oren Weimann. Binary jumbled pattern matching on trees and tree-like structures. Algorithmica, 73(3):571-588, 2015.

[21] Emanuele Giaquinta and Szymon Grabowski. New algorithms for binary jumbled pattern matching. Inf. Process. Lett., 113(14-16):538-542, 2013.

[22] Idrissa Kaboré and Boucaré Kientéga. Abelian complexity of Thue-Morse word over a ternary alphabet. In Proc. of the 11th Int. Conf. on Combinatorics on Words WORDS 2017, volume 10432 of LNCS, pages 132-143. Springer, 2017.

[23] M. Lothaire. Algebraic Combinatorics on Words. Cambridge Univ. Press, 2002.

[24] Blake Madill and Narad Rampersad. The abelian complexity of the paperfolding word. Discrete Mathematics, 313(7):831-838, 2013. doi:10.1016/j.disc.2013.01.005.

[25] Tanaeem M. Moosa and M. Sohel Rahman. Sub-quadratic time and linear space data structures for permutation matching in binary strings. J. Discr. Alg., 10:5-9, 2012.

[26] Giuseppe Pirillo. Inequalities characterizing standard sturmian and episturmian words. Theor. Comput. Sci., 341(1-3):276-292, 2005. doi:10.1016/j.tcs.2005.04.008.

[27] Gwénaël Richomme, Kalle Saari, and Luca Q. Zamboni. Abelian complexity of minimal subshifts. J. London Math. Society, 83(1):79-95, 2011. doi:10.1112/jlms/jdq063.

[28] Frank Ruskey, Carla Savage, and T.M.Y. Wang. Generating necklaces. J. Algorithms, 13(3):414-430, 1992. 
[29] Frank Ruskey, Joe Sawada, and Aaron Williams. Binary bubble languages and cool-lex order. J. Comb. Theory, Ser. A, 119(1):155-169, 2012.

[30] Joe Sawada and Aaron Williams. Efficient oracles for generating binary bubble languages. Electr. J. Comb., 19(1):P42, 2012.

[31] Joe Sawada, Aaron Williams, and Dennis Wong. Inside the Binary Reflected Gray Code: Flip-Swap languages in 2-Gray code order. Unpublished manuscript, 2017.

[32] Rani Siromoney, Lisa Mathew, V.R. Dare, and K.G. Subramanian. Infinite Lyndon words. Inf. Proc. Letters, 50:101-104, 1994.

[33] N. J. A. Sloane. The On-Line Encyclopedia of Integer Sequences. Available electronically at http://oeis .org.

[34] Ondrej Turek. Abelian complexity of the Tribonacci word. J. of Integer Sequences, 18, 2015. 